Published in final edited form as:

Neuromodulation. 2018 June ; 21(4): 340-347. doi:10.1111/ner.12699.

\title{
Field distribution of transcranial static magnetic stimulation in realistic human head model
}

\author{
Joseph J. Tharayil ${ }^{1}$, Stefan M. Goetz, PhD ${ }^{2,3}$, John M. Bernabei, BSE ${ }^{4,5}$, and Angel V. \\ Peterchev, PhD $1,2,3,6, \#$ \\ ${ }^{1}$ Department of Biomedical Engineering, Duke University, Durham, NC, USA \\ ${ }^{2}$ Department of Psychiatry and Behavioral Sciences, Duke University, Durham, NC, US \\ ${ }^{3}$ Department of Electrical and Computer Engineering, Duke University, Durham, NC, US \\ ${ }^{4}$ School of Medicine, University of Pennsylvania, Philadelphia, PA, USA \\ ${ }^{5}$ School of Engineering and Applied Sciences, University of Pennsylvania, Philadelphia, PA, USA \\ ${ }^{6}$ Department of Neurosurgery, Duke University, Durham, NC, US
}

\section{Abstract}

Objectives-The objective of this work was to characterize the magnetic field (B-field) that arises in a human brain model from the application of transcranial static magnetic field stimulation (tSMS).

Materials and Methods-The spatial distribution of the B-field magnitude and gradient of a cylindrical, $5.08 \mathrm{~cm} \times 2.54 \mathrm{~cm} \mathrm{NdFeB}$ magnet were simulated in air and in a human head model using the finite element method and calibrated with measurements in air. The B-field was simulated for magnet placements over prefrontal, motor, sensory, and visual cortex targets. The impact of magnetic susceptibility of head tissues on the B-field was quantified.

Results-Peak B-field magnitude and gradient respectively ranged from 179-245 mT and from 13.3-19.0 T/m across the cortical targets. B-field magnitude, focality, and gradient decreased with magnet-cortex distance. The variation in B-field strength and gradient across the anatomical targets largely arose from the magnet-cortex distance. Head magnetic susceptibilities had negligible impact on the B-field characteristics. The half-maximum focality of the tSMS B-field ranged from $7-12 \mathrm{~cm}^{3}$.

\footnotetext{
\#Corresponding author.

Conflict of Interest: J. J. Tharayil and J. M. Bernabei declare no relevant disclosures. S. M. Goetz is inventor on patents and patent applications on conventional transcranial magnetic stimulation with pulsed fields (TMS), and has received royalties through his current and previous employers as well as patent application support and research funding from Magstim. Unrelated to brain stimulation, he is also employed part-time by Porsche AG, Stuttgart, Germany. A. V. Peterchev is inventor on patents and patent applications and has received research and travel support as well as patent royalties from Rogue Research, research and travel support, consulting fees, as well as equipment loan from Tal Medical, patent application support and hardware donations from Magstim, as well as equipment loans from MagVenture, all related to technology for conventional TMS with pulsed fields.

Authorship statement: A.V. Peterchev designed the study. S.M. Goetz conducted field measurements and set up the magnet model. J.J. Tharayil and J.M. Bernabei conducted the field simulations. J.J. Tharayil analyzed the data and prepared the figures. All authors wrote and approved the final manuscript.
} 
Significance-This is the first presentation and characterization of the 3D spatial distribution of the B-field generated in a human brain model by tSMS. These data can provide quantitative dosing guidance for tSMS applications across various cortical targets and subjects. The finding that the Bfield gradient is high near the magnet edges should be considered in studies where neural tissue is placed close to the magnet. The observation that susceptibility has negligible effects confirms assumptions in the literature.

\section{Keywords}

Transcranial; static magnetic field; neuromodulation; tSMS; model

\section{Introduction}

There is accumulating evidence that transcranial stimulation with sufficiently strong and sustained static magnetic field results in alterations of brain function. In humans, neuromodulatory effects have been reported after transcranial static magnetic field stimulation (tSMS) of primary motor (1-3), supplementary motor $(3)$, visual $(4,5)$, sensorimotor (6), and primary somatosensory (7) cortices. As well, effects of a static magnetic field on the state of neural tissue have been demonstrated in rat brain slices (8), in vitro preparations of rat GH3 cells (9), rat in vivo (10), mouse pyramidal neurons (11), Guinea pig spinal cord (12), Drosophila motor neuron (13), and rat trigeminal root ganglion (14). These effects, combined with the exceptional simplicity of tSMS hardware, confer potential for therapeutic applications in psychiatry and neurology $(15,16) .1$

The mechanism for the effects of static magnetic fields on neural tissue remains unknown, although various possibilities have been discussed, including deformation of ion channels due to reorientation of diamagnetic anisotropic plasma membrane phospholipids (17), coupling of mechanically-activated ion channels to ferromagnetic particles (18), and activation of voltage-gated channels by the Hall effect $(19,20)$. Nevertheless, both theoretical considerations and experimental results indicate that the magnetic dose (i.e., the characteristics of the magnetic flux density, or B-field) has a significant impact on response $(1,10)$.

In order to provide proper B-field dosimetry for experimental studies, match dosing across experimental systems, and enable investigations of tSMS mechanisms, it is necessary to know the 3D spatial characteristics of the B-field generated by tSMS magnets. At present, there is only basic characterization of the tSMS B-field, comprising magnitude measurements at several points in space $(3,21)$. It is important to extend these data to provide a high-resolution B-field distribution at the spatial scales commensurate with the size of neurons and neuron populations, and inclusive of various distances and locations of experimental systems relative to the magnet. Furthermore, while the effects of magnetic susceptibility of biological tissues are expected to be small, their influence on the B-field strength and gradient has not been quantified.

\footnotetext{
${ }^{1}$ Sensitivity of neurons to magnetic fields can also be engineered using genetically modified ion channels—a novel and promising approach called magneto-genetics $(38,39)$.
} 
Computational models of the 3D electric field distribution induced by the time-varying magnetic field of conventional transcranial magnetic stimulation (TMS) are recognized as an important dosing and analysis tool (22-25). Unlike conventional TMS, which is understood to act via its electric field, tSMS appears to act via its static magnetic field. Therefore, it is compelling to apply computational modeling to determine the full 3D distribution of the tSMS magnetic field, analogously to the electric field in TMS. This requires development of appropriate models of the tSMS permanent magnet (as opposed to TMS electromagnetic coil) and human head with magnetic (versus electric) properties of the various tissues.

Addressing this need, in this paper, we present 3D simulations of the B-field of a magnet commonly used in tSMS, including field distributions in air and in a computational model of the human head for several magnet placements, as well as quantification of the effect of head tissue magnetic susceptibility.

\section{Methods}

\subsection{Magnet description}

We characterized a permanent magnet typically used for tSMS $(1-4,6,26,27)$. The magnet is made of grade N52 NdFeB alloy and is cylindrical with diameter of 2" $(5.08 \mathrm{~cm})$ and height of 1” (2.54 cm) (Model \# ND076-N52, Applied Magnets, Plano, TX; see Fig. 1).

\subsection{B-field measurement}

The actual B-field strength at several distances along the central axis of the magnet was measured with a Tesla-meter (Model 5080, F. W. Bell, Milwaukie, OR) positioned using a micrometer. The measurement error of the micrometer was estimated to be $\pm 0.125 \mathrm{~mm}$ and the measurement error of the B-field was obtained from the Tesla-meter manual, as plotted in Fig. 3b. These measurements were used to calibrate the computational B-field model by scaling the remanent magnetization to match that of the physical magnet, as described below.

\subsection{Magnet model}

All simulations were conducted in COMSOL v5.2a (COMSOL, Inc., Burlington, MA), using the "Magnetic Fields, No Currents" module and the conjugate gradient iterative solver with relative tolerance of 0.001 . This module was chosen since the tSMS magnetic field is static with respect to the subject's head, and therefore no electric currents are induced. In simulations without the head, the magnet was surrounded by an air sphere with diameter 40 $\mathrm{cm}$, centered on the magnet. In simulations with the head, the head and magnet were surrounded by an air sphere with diameter $40 \mathrm{~cm}$, centered on the head. In both cases, the surface of the sphere was defined as a magnetic insulation boundary condition, corresponding to the B-field being tangential to the boundary surface. A diameter of $40 \mathrm{~cm}$ was chosen since this provided $>8 \mathrm{~cm}$ from the magnet surface to the bounding sphere and our physical measurements showed that the B-field is very small at this distance (see Fig. 3). The model was defined as follows 


$$
\begin{aligned}
& \boldsymbol{B}=\mu_{0} \boldsymbol{\mu}_{\boldsymbol{r}}(\boldsymbol{H}) \boldsymbol{H}+\boldsymbol{B}_{\boldsymbol{r}}, \text { for the magnet, and } \\
& \boldsymbol{B}=\mu_{0} \mu_{r} \boldsymbol{H}, \text { for all other materials }
\end{aligned}
$$

where $\boldsymbol{B}$ is the magnetic flux density, $\boldsymbol{H}$ is the magnetic field strength, $\boldsymbol{B}_{\boldsymbol{r}}$ is the remanent magnetic flux density of the magnet, $\mu_{0}$ is the magnetic permeability of free space, and $\mu_{r}$ is the relative magnetic permeability of each material, which is equal to $1+\chi_{V}$ where $\chi_{V}$ is the magnetic susceptibility of the material. The relative permeability of the magnet was defined as (28)

$$
\boldsymbol{\mu}_{\boldsymbol{r}}(\boldsymbol{H})=\left(\begin{array}{ccc}
\mu_{r \perp} & 0 & 0 \\
0 & \mu_{r \perp} & 0 \\
0 & 0 & \mu_{r \|}\left(H_{z^{\prime}}\right.
\end{array}\right)
$$

where $\mu_{r \perp}$ and $\mu_{t||}\left(H_{z^{\prime}}\right)$ are, respectively, the relative permeabilities perpendicular and parallel to the direction of magnetization along the magnet central axis, $Z^{\prime}$. Initial values for the magnetic scalar potential, used to compute $\boldsymbol{H}$, were set to zero.

A finite element model of the tSMS magnet was implemented to include (1) a non-linear magnetic B-H curve reflecting the properties of the N52 NdFeB compound as measured by the supplier (VAC Magnetics LLC, Elizabethtown, KY), (2) accurate geometry reflecting the actual magnet cylindrical shape, dimensions, and beveled edges, and (3) high-density finite element mesh, with approximately 370,000 and 7,000 tetrahedral elements respectively in the models of the stand-alone magnet and its combination with the head (this reduction of the magnet model resolution resulted in a mean error of only $1.1 \times 10^{-4} \mathrm{~T}$ ). The magnet model in simulations without the head was constructed within COMSOL, while the magnet model used in simulations with the head was created in Simpleware. The remanent flux density $\boldsymbol{B}_{\boldsymbol{r}}$ of the magnet material was set to have direction corresponding to the magnetization along the magnet central axis $\left(z^{\prime}\right)$ and magnitude of $1.29 \mathrm{~T}$ according to the supplier-provided B-H curve (Fig. 1) adjusted to match the measurements. Parameter $\mu_{r \perp}$ was set to a constant of 1.17 (28) and $\mu_{t||}\left(H_{Z^{\prime}}\right)$ was obtained by taking the derivative $\Delta B / \Delta H$ of the B-H curve (Fig. 1).

\subsection{Human head model}

An anatomically accurate head model derived from MRI data (Fig. 2a-b) was used to characterize the B-field generated by tSMS in the human brain. The model's generation and structure were reported previously (24). Briefly, the model was constructed from T1weighted MRI ( $1 \mathrm{~mm} \times 1 \mathrm{~mm} \times 1 \mathrm{~mm}$ resolution) of a healthy 34-year-old male human subject. The magnet model described above was positioned relative to the head model in Simpleware, and the combined head-and-magnet model was imported into COMSOL from Simpleware as a mesh. The head model mesh had $\sim 6$ million tetrahedral elements. The magnetic susceptibility values used for the various compartments in the head model are summarized in Table 1. Susceptibility of scalp was assumed to be equal to that of fat, due to 
unavailability of measurements for skin. Since a static magnetic field is simulated, the electric properties of head tissues (conductivity and permittivity) are irrelevant.

\subsection{B-field characterization}

First, the magnet was simulated surrounded by air in COMSOL, without the head model present. Second, the magnet was placed over various targets in the left hemisphere of the head model, including the dorsolateral prefrontal cortex (DLPFC), hand knob of the primary motor cortex (M1), sensorimotor cortex (SMC), and occipital cortex. The magnet position was selected for the DLPFC, M1, and occipital cortex with reference to an atlas (29). The magnet position for SMC was selected with reference to the standard EEG electrode placement system (3). All targets were simulated for two distances of the magnet surface from the underlying scalp $-0 \mathrm{~mm}$ and $5 \mathrm{~mm}-$ the latter representing the case when hair or hardware are interposed between the magnet and the scalp.

The spatial distribution of the B-field magnitude and gradient were reported, since they may both contribute to effects on neural tissue. The magnitude of the B-field gradient was computed in COMSOL, by taking the Frobenius norm of the B-field Jacobian matrix. For each anatomical target and magnet position, minimum and maximum B-field in the brain model as well as maximum B-field gradient magnitude were obtained from the built-in COMSOL postprocessing toolbox, which finds extrema over the entirety of a defined volume. Analogous to characterization of the electric field focality in TMS $(23,25,31)$, the B-field focality in tSMS was characterized by the volume of the brain model with B-field above half the maximum value in the brain model. Similarly, the focality of the B-field gradient was characterized by the volume of the brain model with B-field gradient above half the maximum value of the gradient in the brain model. All focality metrics were computed in COMSOL by integrating over the sub-volume of the brain model where the Bfield magnitude (or B-field gradient magnitude) was greater than half of its peak value. Magnet-to-cortex distance was defined as the distance between the location of the peak Bfield in the grey matter and the nearest point on the magnet, and was computed in COMSOL.

To determine the influence of the magnetic susceptibility of biological tissues, the simulation results from models with the magnet flush with the scalp were compared between the cases when appropriate susceptibility values were assigned to the head model compartments versus assigning all compartments to the susceptibility of air.

Data analysis was conducted in MATLAB (The Mathworks, Natick, MA). For comparisons between the models with and without tissue susceptibility, data points were selected automatically according to the geometry of the model, so grid size varied; minimum point spacing ranged from $0.13-0.58 \mathrm{~mm}$ depending on direction. Regression lines were computed with the Statistics Toolbox, using ordinary least squares. 


\section{Results}

\subsection{B-field characteristics in air}

Along the central axis of the magnet, B-field magnitudes decreased exponentially with distance (Fig. 3a-b) with a peak magnitude of $443 \mathrm{mT}$, dropping to $159 \mathrm{mT}$ at a distance of $2 \mathrm{~cm}$ from the base of the magnet. The B-field values from the calibrated simulation were in agreement with the corresponding measured values, with a mean relative error of $1.28 \%$ (Fig. 3b). The highest spatial gradient in the magnetic field, up to $1.12 \times 10^{6} \mathrm{~T} / \mathrm{m}$, was observed near the edges of the magnet (Fig. 3c).

\subsection{B-field characteristics in human head model}

The B-field generated in the head model for several magnet positions is shown in Fig. 4 and characterized in Fig. 5. Full 3D distributions of the B-field magnitude and gradient are available in Supplementary Videos S1-S4 and S5-S8, respectively. The B-field in the brain model ranged from 0.778 to $245 \mathrm{mT}$ for the $0 \mathrm{~mm}$ scalp magnet spacing, and 0.688 to 180 $\mathrm{mT}$ for the $5 \mathrm{~mm}$ magnet displacement. As expected, the distance between the magnet and the cortical target largely determined the peak B-field strength in the cortex $\left(R^{2}=0.99, p=\right.$ $1.14 \times 10^{-6}$; Fig. 6a). This relationship was nearly linear since the range of distances was narrow $(1-2 \mathrm{~cm})$ and corresponded to an approximately linear section of the curve in Fig. 3b. Placing the magnet $5 \mathrm{~mm}$ from the scalp decreased the peak B-field strength at the target by $25.0 \%$ on average.

Magnet placement had significant effects on focality as well (Fig. 5b). For $5 \mathrm{~mm}$ compared to $0 \mathrm{~mm}$ displacement, the half-maximum volume of the B-field magnitude increased by $7.5 \%$ on average, indicating reduction of focality. The B-field was most focal for placement over SMC and least focal for placement over the occipital cortex, with a difference of 40$52 \%$. Since the dependence of focality on the specific cortical target was different from the dependence of the peak B-field magnitude, it appears that the local cortical geometry affected these two metrics differently.

The peak spatial gradient of the B-field ranged from $13.3 \mathrm{~T} / \mathrm{m}$ to $19.0 \mathrm{~T} / \mathrm{m}$ for the $0 \mathrm{~mm}$ scalp magnet spacing (see Fig. 5c). For all targets, the peak spatial gradient of the B-field was higher for magnet placements flush with the scalp, with an average decrease of $28.8 \%$ for placement $5 \mathrm{~mm}$ away from the scalp. As with the magnitude of the B-field, the distance between the magnet and the cortical target largely determined peak gradient in the cortex $\left(R^{2}=0.99, p=8.8 \times 10^{-7} ;\right.$ Fig. 6b). The focality of the B-field spatial gradient (Fig. $5 \mathrm{~d}$ ) varied with magnet placement with a dependence similar to that of the B-field magnitude focality.

\subsection{Effect of head magnetic susceptibility}

The magnetic susceptibility of head tissues had a negligible effect on the B-field magnitude or gradient. As summarized in Table 2, all differences were well below 1\%. The maximum absolute differences occurred between 0 and $2.5 \mathrm{~cm}$ from the respective sites of peak B-field magnitude or gradient. 


\section{Discussion}

Our characterization of the tSMS B-field was consistent with and extended previous measurements $(3,21)$ by providing a complete $3 \mathrm{D}$ distribution of the field magnitude and gradient in air and in a computational model of the human head and brain. At distances up to approximately $4.7 \mathrm{~cm}$ from the base of the magnet, the B-field was $>40 \mathrm{mT}$, which is believed to be sufficient for physiological effects (32).

The focality of the tSMS B-field was characterized by a half-maximum volume in the 7-12 $\mathrm{cm}^{3}$ range. In comparison, the half-maximum volume of the electric field induced by figure-8 TMS coils with $60-70 \mathrm{~mm}$ loop diameter, conventionally used in humans, is estimated to be $17-21 \mathrm{~cm}^{3}$ and $\sim 80 \mathrm{~cm}^{3}$ in spherical (25) and anatomically-realistic (31) head models, respectively. Thus, the B-field generated by tSMS in humans with the magnet we modeled is more focal than the E-field induced in conventional TMS.

The choice of anatomical target and the distance from the magnet to the scalp both had a significant effect on the B-field strength and focality, driven largely by the magnet-to-cortex distance. This has several implications: First, for maximum efficiency of stimulation, the distance from the magnet to the scalp should be minimized. Second, when tSMS is applied over various brain targets, the magnet-to-cortex distance should be considered. Third, since both magnet-to-scalp and scalp-to-cortex distances may vary across individuals due to presence of hair and differences in head size and anatomy, the magnet-to-cortex distance may need to be adjusted to compensate for this individual variability and deliver fixed Bfield strength at the brain target. Note that this is less straightforward than in conventional TMS, which utilizes electronic control of the pulse intensity and has available a simple motor readout of brain activation. In contrast, tSMS B-field strength control with a permanent magnet may involve mechanically adjustable positioning of the magnet relative to the head, and may have to rely on measures of scalp-to-cortex distance obtained from imaging.

Our simulations revealed significant edge effects in the spatial gradient of the B-field. Close to the magnet, the peak spatial gradient was highest at the corners of the magnet, but at greater distances, the location of the peak spatial gradient was shifted inward, such that in the cortex, peak spatial gradient is directly below the center of the magnet. This is of particular importance to studies where neural tissue is very close to the magnet, such as in our previously reported in vitro brain slice studies (8). A high spatial gradient may be important for two reasons. First, it increases the sensitivity of the B-field exposure to the exact placement of the magnet relative to the target. Second, the gradient may contribute to the biological effects of the B-field.

As the magnetic susceptibility of tissues had a negligible impact on the B-field characteristics, our results provide quantitative justification for the assumption that measurements of B-fields taken in air do not need to be adjusted for in-vivo applications (27). Also for this reason, uncertainties in the tissue susceptibility estimates or the inclusion of more tissues is unlikely to change significantly our results. For example, even though hemoglobin contains iron, this ionic iron is not in the ferromagnetic grid order and electron 
configuration; consequently, the magnetic susceptibility of arterial and venous blood is only $-9 \mathrm{ppm}$ and $-8 \mathrm{ppm}$, respectively (33). These are on the same order of magnitude as the magnetic susceptibilities of the tissues we modeled (see Table 1). Since the head tissues do not affect significantly the B-field, the effect of geometric uncertainties such as in the magnet placement can be easily estimated from the data we provide, by considering the Bfield magnitude or gradient change for a given change in distance.

Finally, we simulated only one magnet geometry and size, typical for tSMS, but the presented simulation methods can be applied to any magnet configuration.

\section{Conclusion}

The presented 3D distribution of the B-field magnitude and spatial gradient provides dosimetric information for a typical tSMS magnet. The same methods can be used to characterize other magnet types. A high-resolution map of the B-field is important for proper analysis of experimental data, study of mechanisms, and matching of the dose across species and experimental preparations. Potentially physiologically relevant B-field magnitudes extend several centimeters into the brain. The B-field focality was higher than that of the induced electric field of conventional figure-8 TMS coils. The most significant contributor to variation in B-field strength and focality was distance to the cortex, which is determined by the magnet placement and individual anatomy. The magnetic field gradient varied with distance and position relative to the magnet as well, and was strongest around the magnet edges, which may be important for experimental preparations that are placed very close to the magnet. Finally, we confirmed that the magnetic susceptibility of tissues has negligible effects on the B-field characteristics.

\section{Supplementary Material}

Refer to Web version on PubMed Central for supplementary material.

\section{Acknowledgments}

Financial support: This work was supported in part by a Research Incubator Award from Duke Institute for Brain Sciences. The human head model was created with funding by NIH grant R01MH091083. The authors would like to thank Gabriel M. Kroch, Dr. Zhi-De Deng, and Dr. Won Hee Lee for technical assistance and contributions to earlier versions of this report, Dr. Roland Zoller from VAC Magnetics for providing magnetic characterization of the N52 material, and Dr. Elizabeth Bucholz and Dr. Chunlei Liu for information on tissue susceptibility. Some preliminary results of this study were presented at the 37th International Conference of the IEEE Engineering in Medicine and Biology Society, Milan, Italy, August 25-29, 2015.

\section{References}

1. Oliviero A, Mordillo-Mateos L, Arias P, Panyavin I, Foffani G, Aguilar J. Transcranial static magnetic field stimulation of the human motor cortex. J Physiol. 2011; 589(Pt 20):4949-4958. [PubMed: 21807616]

2. Silbert BI, Pevcic DD, Patterson HI, Windnagel KA, Thickbroom GW. Inverse correlation between resting motor threshold and corticomotor excitability after static magnetic stimulation of human motor cortex. Brain Stimul. 2013; 6(5):817-820. [PubMed: 23598254]

3. Kirimoto H, Asao A, Tamaki H, Onishi H. Non-invasive modulation of somatosensory evoked potentials by the application of static magnetic fields over the primary and supplementary motor cortices. Sci Rep. 2016; 6:34509. [PubMed: 27698365] 
4. Gonzalez-Rosa JJ, Soto-Leon V, Real P, Carrasco-Lopez C, Foffani G, Strange BA, et al. Static magnetic field stimulation over the visual cortex increases alpha oscillations and slows visual search in humans. J Neurosci. 2015; 35(24):9182-9193. [PubMed: 26085640]

5. Aguila J, Cudeiro J, Rivadulla C. Effects of static magnetic fields on the visual cortex: reversible visual deficits and reduction of neuronal activity. Cereb Cortex. 2016; 26(2):628-638. [PubMed: 25260705]

6. Kirimoto H, Tamaki H, Matsumoto T, Sugawara K, Suzuki M, Oyama M, et al. Effect of transcranial static magnetic field stimulation over the sensorimotor cortex on somatosensory evoked potentials in humans. Brain Stimul. 2014; 7(6):836-840. [PubMed: 25444588]

7. Carrasco-Lopez C, Soto-Leon V, Cespedes V, Profice P, Strange BA, Foffani G, et al. Static magnetic field stimulation over parietal cortex enhances somatosensory detection in humans. J Neurosci. 2017

8. Klein, RC., Goetz, SM., Liedtke, WB., Moore, SD., Peterchev, AV. Static magnetic field modulates excitatory activity in layer II/III pyramidal neurons of the rat motor cortex; 2013 6th International IEEE/EMBS Conference on Neural Engineering (NER); 2013. p. 1190-1193.

9. Rosen AD. Effect of a $125 \mathrm{mT}$ static magnetic field on the kinetics of voltage activated $\mathrm{Na}+$ channels in GH3 cells. Bioelectromagnetics. 2003; 24(7):517-523. [PubMed: 12955757]

10. Houpt TA, Cassell JA, Cason AM, Riedell A, Golden GJ, Riccardi C, et al. Evidence for a cephalic site of action of high magnetic fields on the behavioral responses of rats. Physiol Behav. 2007; 92(4):665-674. [PubMed: 17568635]

11. Li G, Cheng L, Qiao X, Lin L. Characteristics of delayed rectifier potassium channels exposed to 3 mT static magnetic field. IEEE Trans Magn. 2010; 46(7):2635-2638.

12. Coots A, Shi R, Rosen AD. Effect of a 0.5-T static magnetic field on conduction in guinea pig spinal cord. J Neurol Sci. 2004; 222(1-2):55-57. [PubMed: 15240196]

13. Giachello CNG, Scrutton NS, Jones AR, Baines RA. Magnetic fields modulate blue-lightdependent regulation of neuronal firing by cryptochrome. J Neurosci. 2016; 36(42):10742-10749. [PubMed: 27798129]

14. Shen JF, Chao YL, Du L. Effects of static magnetic fields on the voltage-gated potassium channel currents in trigeminal root ganglion neurons. Neurosci Lett. 2007; 415(2):164-168. [PubMed: 17289262]

15. Paulus W. Transcranial static magnetic field stimulation in man: making things as simple as possible? J Physiol. 2011; 589(Pt 24):5917-5918. [PubMed: 22025669]

16. Bestmann S. Neurostimulation: A new way to influence cortical excitability? Curr Biol. 2011; 21(21):R893-R894. [PubMed: 22075430]

17. Rosen AD. Mechanism of action of moderate-intensity static magnetic fields on biological systems. Cell Biochem Biophys. 2003; 39(2):163-173. [PubMed: 14515021]

18. Dobson J, St Pierre T. Application of the ferromagnetic transduction model to D.C. and pulsed magnetic fields: effects on epileptogenic tissue and implications for cellular phone safety. Biochem Biophys Res Commun. 1996; 227(3):718-723. [PubMed: 8886000]

19. Balcavage XW, Alvager T, Swez J, Goff WC, Fox TM, Abdullyava S, et al. A mechanism for action of extremely low frequency electromagnetic fields on biological systems. Biochem Biophys Res Commun. 1996; 222(2):374-378. [PubMed: 8670212]

20. St Pierre TG, Dobson J. Theoretical evaluation of cell membrane ion channel activation by applied magnetic fields. Eur Biophys J. 2000; 29(6):455-456. [PubMed: 11081406]

21. Rivadulla C, Foffani G, Oliviero A. Magnetic field strength and reproducibility of neodymium magnets useful for transcranial static magnetic field stimulation of the human cortex. Neuromodulation. 2014; 17(5):432-438.

22. Wagner T, Valero-Cabre A, Pascual-Leone A. Noninvasive human brain stimulation. Annu Rev Biomed Eng. 2007; 9:527-565. [PubMed: 17444810]

23. Parazzini M, Fiocchi S, Chiaramello E, Roth Y, Zangen A, Ravazzani P. Electric field estimation of deep transcranial magnetic stimulation clinically used for the treatment of neuropsychiatric disorders in anatomical head models. Med Eng Phys. 2017; 43:30-38. [PubMed: 28236602] 
24. Lee WH, Lisanby SH, Laine AF, Peterchev AV. Comparison of electric field strength and spatial distribution of electroconvulsive therapy and magnetic seizure therapy in a realistic human head model. Eur Psychiatry. 2016; 36:55-64. [PubMed: 27318858]

25. Deng ZD, Lisanby SH, Peterchev AV. Electric field depth-focality tradeoff in transcranial magnetic stimulation: Simulation comparison of 50 coil designs. Brain Stimul. 2013; 6(1):1-13. [PubMed: 22483681]

26. Oliviero A, Carrasco-Lopez MC, Campolo M, Perez-Borrego YA, Soto-Leon V, Gonzalez-Rosa JJ, et al. Safety study of transcranial static magnetic field stimulation (tSMS) of the human cortex. Brain Stimul. 2015; 8(3):481-485. [PubMed: 25595064]

27. Rivadulla C, Foffani G, Oliviero A. Magnetic field strength and reproducibility of neodymium magnets useful for transcranial static magnetic field stimulation of the human cortex. Neuromodulation. 2014; 17(5):438-441. [PubMed: 24125470]

28. Chavanne J, Meunier G, Sabonnadihre JC. Nonlinear permanent magnets modelling with the finite element method. IEEE Trans Magn. 1989; 25(5):3581-3583.

29. Nolte, J. The Human Brain: An Introduction to Its Functional Anatomy. Fifth. St. Louis: Mosby, Inc; 2002.

30. Purves, D., Augustine, G., Fitzpatrick, D., Hall, W., Lamantia, AS., White, L. Neuroscience. Fifth. Sunderland, MA: Sinauer Associates; 2012.

31. Guadagnin V, Parazzini M, Fiocchi S, Liorni I, Ravazzani P. Deep Transcranial Magnetic Stimulation: Modeling of Different Coil Configurations. IEEE Trans Biomed Eng. 2016; 63(7): 1543-1550. [PubMed: 26560868]

32. Rosen AD. Studies on the effect of static magnetic fields on biological systems. PIERS Online. 2010:133-136.

33. Glaser, R. Biophysics. 5th. Berlin, New York: Springer; 2001.

34. Schenck JF. The role of magnetic susceptibility in magnetic resonance imaging: MRI magnetic compatibility of the first and second kinds. Med Phys. 1996; 23(6):815-850. [PubMed: 8798169]

35. Boesch C, Slotboom J, Hoppeler H, Kreis R. In vivo determination of intra-myocellular lipids in human muscle by means of localized 1H-MR-spectroscopy. Magn Reson Med. 1997; 37(4):484493. [PubMed: 9094069]

36. Buch S, Liu S, Ye Y, Cheng YCN, Neelavalli J, Haacke EM. Susceptibility mapping of air, bone, and calcium in the head. Magn Reson Med. 2015; 73(6):2185-2194. [PubMed: 25046134]

37. Peprah MK, Astary GW, Mareci TH, Meisel MW. Absolute magnetic susceptibility of rat brain tissue. Magn Reson Med. 2014; 72(3):876-879. [PubMed: 24123079]

38. Wheeler MA, Smith CJ, Ottolini M, Barker BS, Purohit AM, Grippo RM, et al. Genetically targeted magnetic control of the nervous system. Nat Neurosci. 2016; 19(5):756-761. [PubMed: 26950006]

39. Stanley SA, Kelly L, Latcha KN, Schmidt SF, Yu X, Nectow AR, et al. Bidirectional electromagnetic control of the hypothalamus regulates feeding and metabolism. Nature. 2016; 531(7596):647-650. [PubMed: 27007848] 


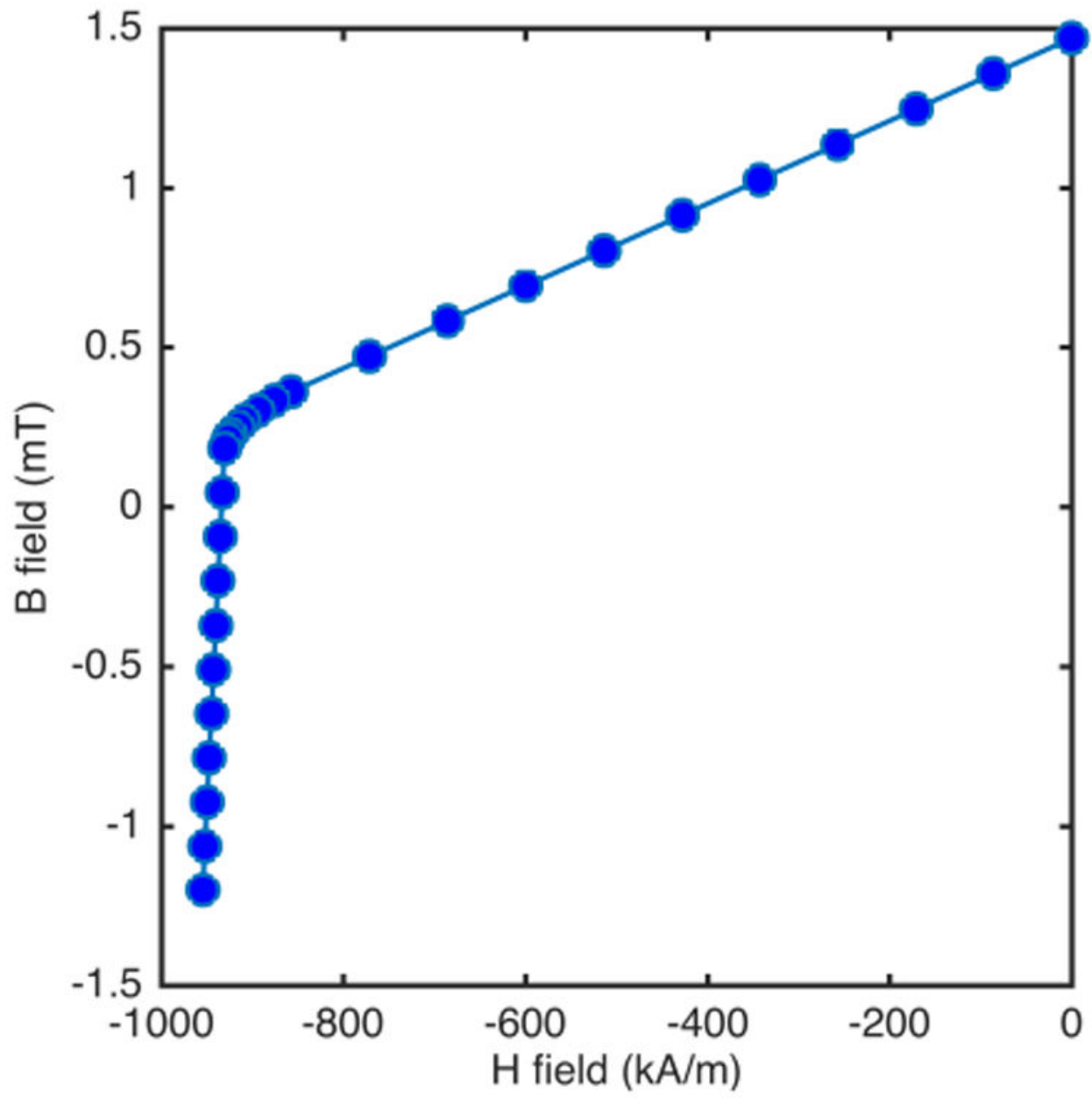

Figure 1.

Magnet material magnetization (B-H) curve. 
a

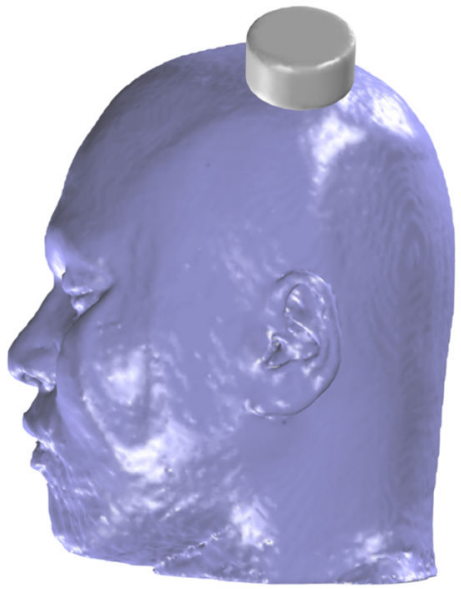

b

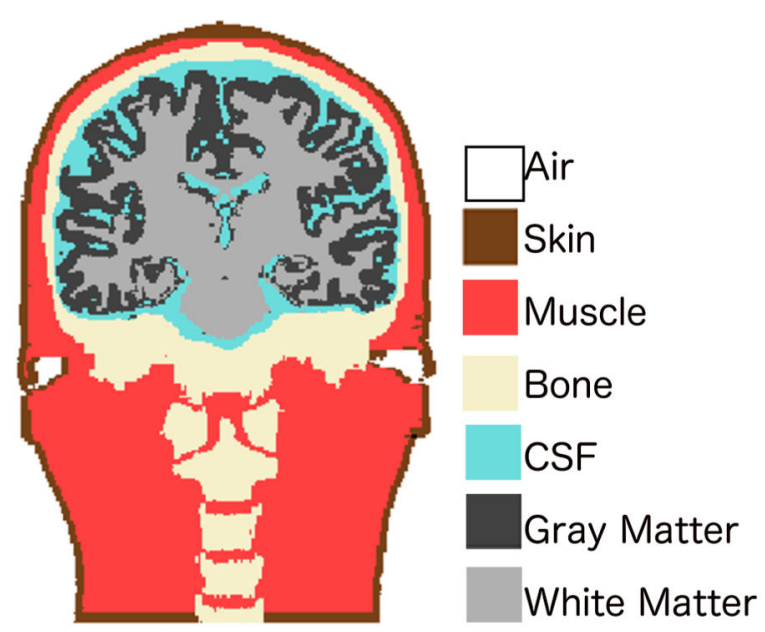

Figure 2.

a. 3D head and magnet model visualization. b. Head model segmentation. Susceptibility values corresponding to the various head compartments are listed in Table 1. 

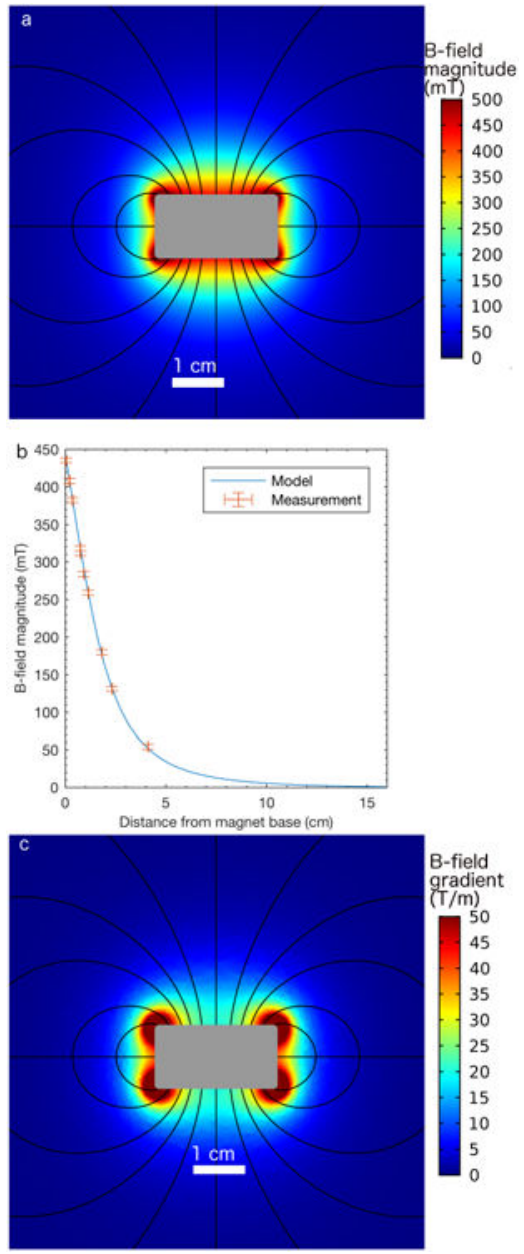

Figure 3 .

Magnetic field characteristics in air. a. B-field magnitude spatial distribution, with magnetic flux lines. b. Comparison of measurement and model of B-field magnitude under the center of the magnet as a function of distance. The estimated measurement error is marked with whiskers. c. B-field spatial gradient distribution, with magnetic flux lines. 

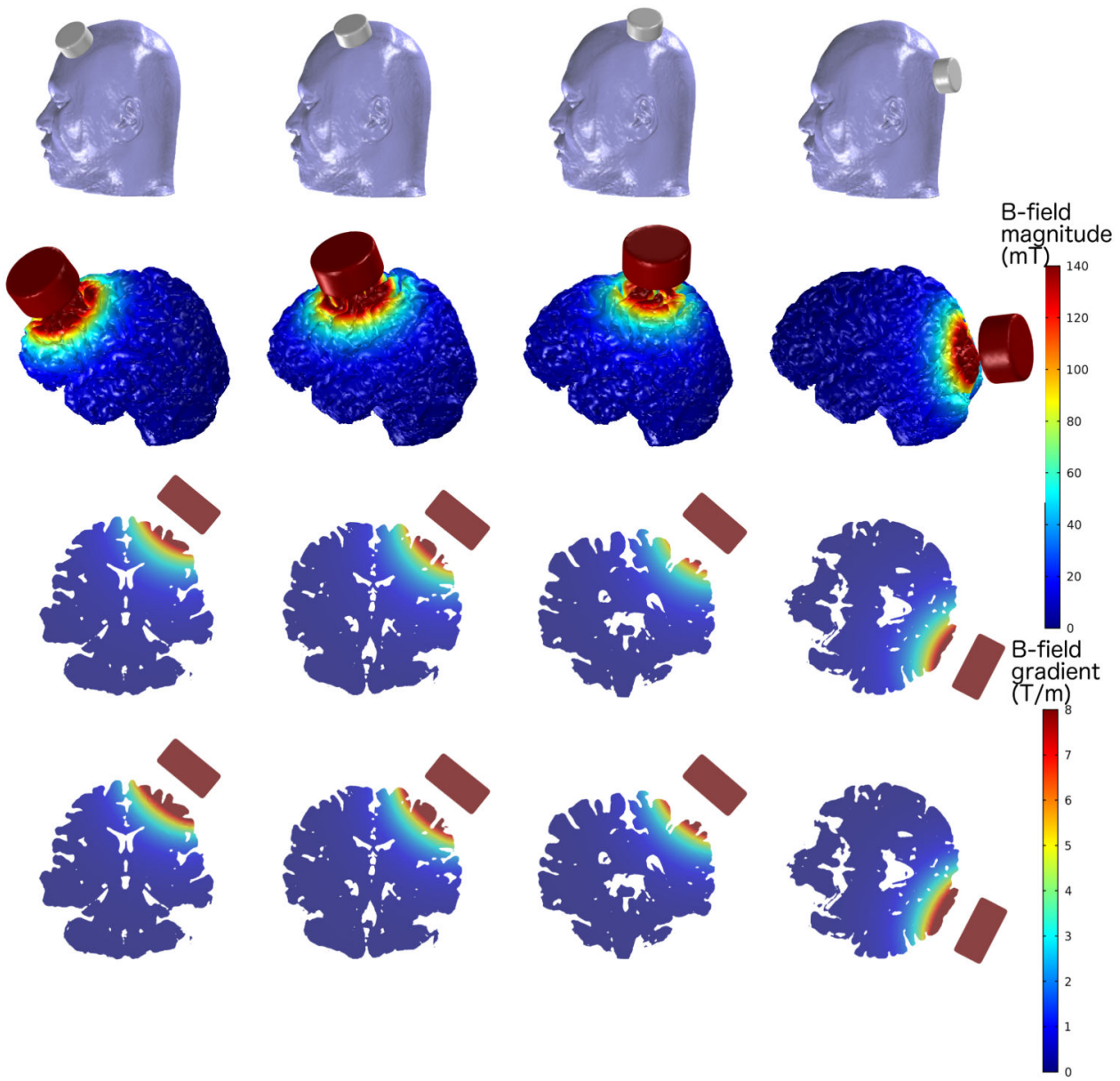

Figure 4.

Simulated tSMS B-field in the brain. Magnet is placed flush with scalp, over the left hemisphere, and over (columns left to right) dorsolateral prefrontal cortex (DLPFC), primary motor cortex (M1), sensorimotor cortex, and occipital cortex. Top row: Visualization of the model. Second row: Magnitude distribution on cortical surface. Third row: Magnitude distribution is representative brain slices centered under the magnet. Bottom row: Spatial gradient distribution in the same slices as in the third row. 
a

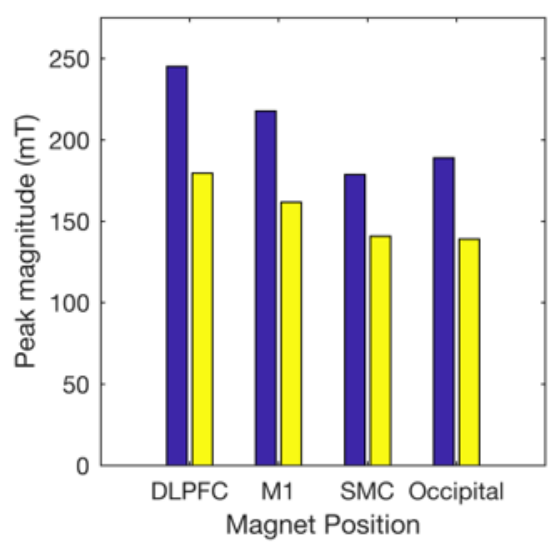

C

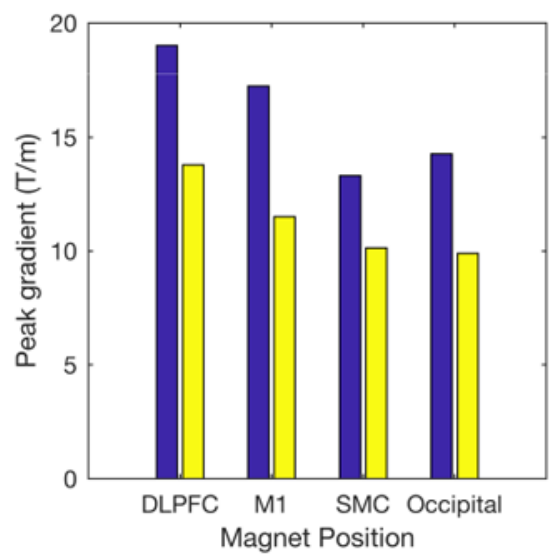

b

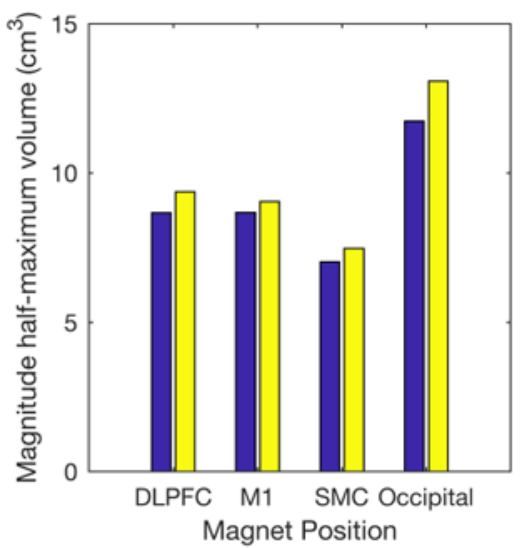

d
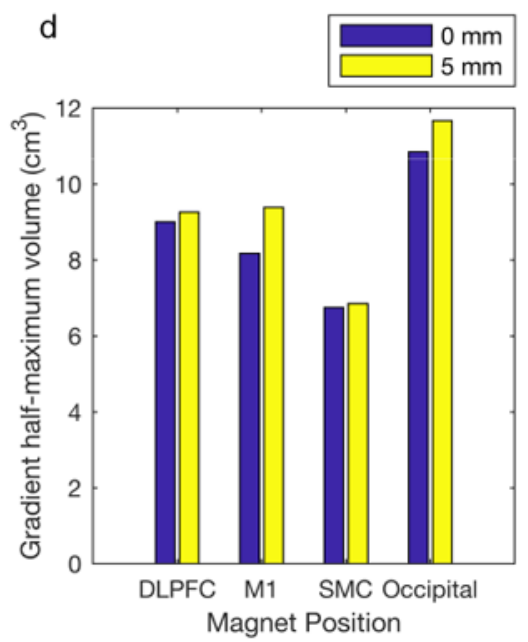

Figure 5.

B-field characterization for the targets depicted in Fig. 4. Peak value (left) and halfmaximum volume (right) of the B-field magnitude (top) and spatial gradient (bottom) in the brain. 

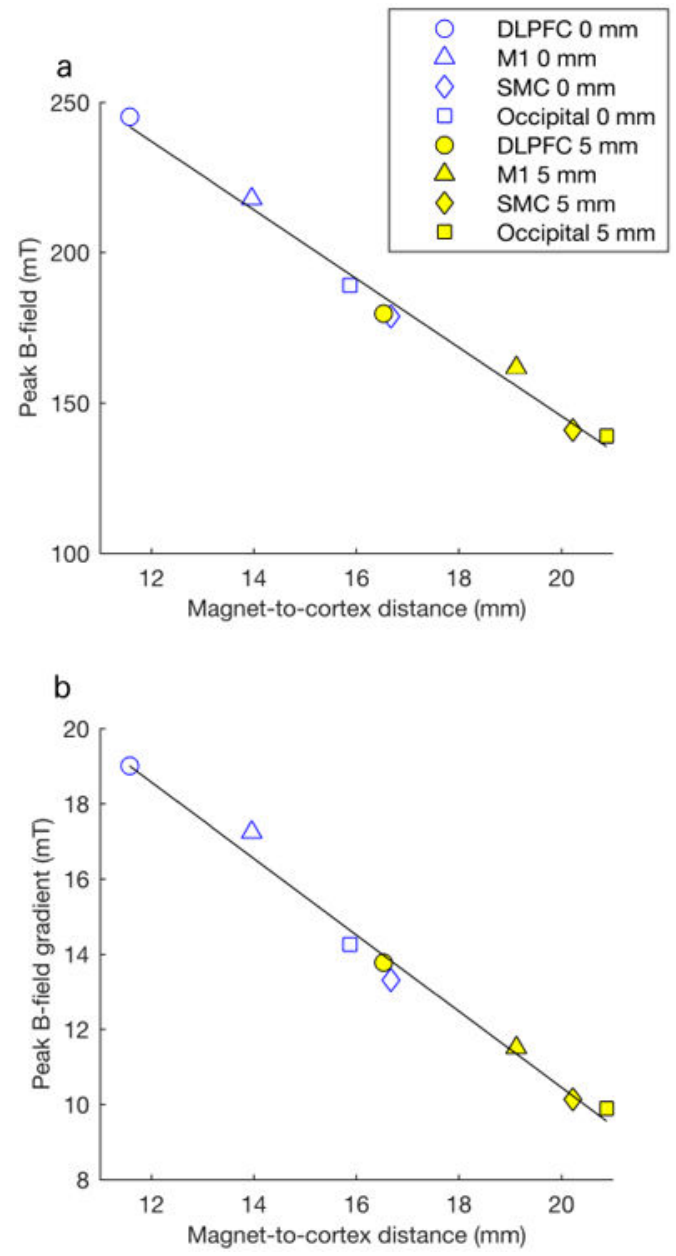

Figure 6.

Dependence of B-field characteristics on distance from cortex. a. Peak B-field magnitude as a function of distance from scalp to cortex. b. Peak B-field gradient as a function of distance from scalp to cortex. Regression lines calculated using ordinary least squares $\left(\mathrm{a}: R^{2}=0.99\right.$, $\left.p<10^{-5} ; \mathrm{b}: R^{2}=0.99, p<10^{-6}\right)$. 


\section{Table 1}

Magnetic susceptibility values for the various compartments of the head model.

\begin{tabular}{|l|l|l|}
\hline Head compartment & Magnetic susceptibility, $\chi_{V}(\mathbf{p p m})$ & Source \\
\hline Air & 0.36 & $(34)$ \\
\hline Skin & -8.44 & $(35)$ \\
\hline Muscle & -9.0 & $(33)$ \\
\hline Bone & -10.94 & $(36)$ \\
\hline CSF & -0.0068 & Dr. Chunlei Liu, Duke University \\
\hline Grey Matter & -9.51 & $(37)$ \\
\hline White Matter & -8.99 & $(37)$ \\
\hline
\end{tabular}




\section{Table 2}

B-field error throughout the brain model for the case when all head tissues were assigned susceptibility of air versus when susceptibilities were assigned according to Table 1 . Mean absolute error was averaged over the whole brain and all magnet placements flush with the scalp. Maximum error is across the brain and magnet placements. B-field vector error was calculated as the magnitude of the vector difference, and therefore captures differences in the field direction as well as magnitude.

\begin{tabular}{|l|l|l|l|}
\hline \multirow{2}{*}{ B-field characteristic } & \multirow{2}{*}{ Mean absolute error } & \multicolumn{2}{|c|}{ Maximum error } \\
\cline { 3 - 4 } & & Absolute & Percentage relative to maximum B field \\
\hline Vector & $5.9 \times 10^{-8} \mathrm{~T}$ & $2.9 \times 10^{-6} \mathrm{~T}$ & $0.0013 \%$ \\
\hline Magnitude & $5.7 \times 10^{-8} \mathrm{~T}$ & $2.7 \times 10^{-6} \mathrm{~T}$ & $0.0013 \%$ \\
\hline Gradient & $4.8 \times 10^{-6} \mathrm{~T} / \mathrm{m}$ & $2.5 \times 10^{-3} \mathrm{~T} / \mathrm{m}$ & $0.0189 \%$ \\
\hline
\end{tabular}

